\title{
Has HCC MET its match?
}

Patients with advanced-stage hepatocellular carcinoma (HCC) have only one treatment option, sorafenib, which is associated with an overall survival of less than a year. Clearly, there is an unmet medical need, but a trial assessing a MET inhibitor seems to offer some hope.

This phase II, double-blind, placebocontrolled clinical trial assessed the oral MET inhibitor tivantinib in patients with advanced-stage HCC and Child-Pugh A cirrhosis who had progressive disease during first-line therapy. The trial was designed to assess time to progression as the primary end point, but crucially it also included an assessment of MET expression in the tumours, using immunohistochemistry. In total, 71 patients received tivantinib and 36 patients were assigned to the placebo arm.

As a whole, the patients in the tivantinib arm did not derive considerable benefit from the therapy- 1.6 months in the therapy arm versus 1.4 months in the placebo arm. Although this improvement in time to progression was statistically significant, it was associated with adverse effects that included grade 3 or worse neutropenia. However, the story was more positive in patients with high expression of MET. In these patients, the median time to progression was 2.7 months and 1.4 months and overall survival was 7.2 months and 3.8 months in the tivantinib arm and placebo arm, respectively.

These results in the subset of patients overexpressing the target protein should be seen as promising, and combinations and phase III trials in these patients will hopefully follow.

\section{Rebecca Kirk}

Original article Santoro, A. et al. Tivantinib for second-line treatment of advanced hepatocellular carcinoma: a randomised, placebo-controlled phase 2 study. Lancet Oncol. doi:10.1016/S1470-2045(12)70490-4

Further reading Peters, S. \& Adjei, A. A. MET: a promising anticancer therapeutic target. Nat. Rev. Clin. Oncol. 9, 314-326 (2012) 